\title{
Vitamine und Spurenelemente in der Onkologie
}

Julian Walter Holch, Marlies Michl, Volker Heinemann, Nicole Erickson

\author{
Vitamine und Spurenelemente werden häufig von Patienten eingenommen, um \\ Tumorerkrankungen vorzubeugen oder deren Fortschreiten zu verhindern. Dies \\ geschieht oft auch ohne Wissen des behandelnden Arztes. Der vorliegende Artikel be- \\ leuchtet die aktuelle Evidenzlage zu den meist untersuchten Mikronährstoffen und leitet \\ hieraus konkrete Handlungsempfehlungen ab.
}

Datenlage Mehrere beobachtende Studien zeigen positive Effekte der Supplementation von Mikronährstoffen in verschiedenen Einsatzbereichen der Onkologie. Oftmals werden diese Studien von Befürwortern einer entsprechenden Herangehensweise zitiert. Beobachtende Studien unterliegen jedoch dem Risiko, beeinflussende Faktoren (engl. biases) nicht adäquat berücksichtigen zu können.

\footnotetext{
Merke

Eine Empfehlung der Supplementation von Mikronährstoffen sollte im Kontext hochwertiger klinischer Studien erwogen werden. Zumeist handelt es sich hierbei um kontrollierte randomisierte Studien.
}

Im Folgenden werden die nach Einschätzung der Autoren wichtigsten Studien zur Supplementation von Mikronährstoffen in der Onkologie dargestellt. Soweit vorhanden werden Metaanalysen unter Beachtung der Qualität der Einzelstudien berücksichtigt. Die Dosierung der angegebenen Mikronährstoffe wird in Prozent der empfohlenen Tagesdosis (engl. Recommended Daily Allowance, RDA) gemäß Richtlinie 2008/100/EG angegeben.

Häufigkeit der Supplementation von Mikronährstoffen Die Angaben dazu, wie häufig onkologische Patienten Mikronährstoffe supplementieren, variieren stark. Je nach Tumorentität, Geschlecht und geografischer Lage nehmen etwa 30 - 90\% der Patienten solche Präparate ein, häufig ohne das Wissen des behandelnden Arztes [1 - 3]. Weiterhin nimmt laut einer Untersuchung jeder zweite Gesunde im Alter über 50 Jahren ergänzend Mikronährstoffe ein [2]. Mit der Supplementation von Mikronährstoffen werden zumeist folgende Ziele verfolgt:

- Vorbeugen onkologischer Erkrankungen

- Vorbeugen und Beheben eines Mikronährstoffmangels bei onkologischen Patienten

- Beeinflussen von Effektivität und Nebenwirkungen onkologischer Therapien

\section{Vorbeugen onkologischer Erkrankungen}

\section{Antioxidantien}

Oxidativer Stress scheint eine wichtige Rolle in der Pathogenese maligner Tumoren zu spielen [4]. Mehrere Beobachtungsstudien zeigen eine signifikant positive Assoziation zwischen einer Ernährung reich an Antioxidantien sowie einem reduzierten Krebsrisiko [5]. Kontrollierte, randomisierte Studien wiesen jedoch überwiegend keinen positiven Effekt durch die gezielte Einnahme von Antioxidantien auf die Mortalität nach [6, 7]. Vielmehr wurde erstmals im Jahr 1994 in der ATBC-Studie offensichtlich, dass die gezielte Supplementation mit Vitamin E $(50 \mathrm{mg} / \mathrm{d}, 417 \% \mathrm{RDA})$ und Beta-Carotin (Provitamin A; $20 \mathrm{mg} / \mathrm{d}, 417 \%$ RDA) nachteilig sein kann [8]:

- In dieser prospektiven Studie wurden 29131 finnische Raucher zwischen Behandlungsarmen mit beiden, einem oder keinem der genannten Antioxidantien randomisiert.

- Wurde Beta-Carotin eingenommen, erhöhte sich die Inzidenz von Lungenkarzinomen signifikant um $18 \%$ sowie die Mortalität um $8 \%$.

Die nachfolgende CARET-Studie zeigte ein vergleichbares Ergebnis, wenn Vitamin A (25.000 IU/d, $938 \%$ RDA) und Beta-Carotin (30 mg/d, 625\% RDA) eingenommen wurden. Der negative Effekt hielt bis zu 6 Jahre nach Ende der Supplementation an [8, 9]. Weiterhin zeigte die SELECT-Studie zur Tumorprävention mit Selen $(200 \mu \mathrm{g} / \mathrm{d}$, $364 \%$ RDA) und Vitamin E (400 IU/d, $3000 \%$ RDA), dass die Supplementation mit Vitamin E das Risiko erhöhte, an einem Prostatakarzinom zu erkranken $[10,11]$. Auch scheint eine hochdosierte Supplementation von Vitamin $\mathrm{E}$ zu einer gesteigerten Gesamtmortalität beizutragen [12]. Bemerkenswerterweise zeigte sich auch ein Nachteil für die gezielte Supplementation für weitere Substanzen wie Vitamin B12 und Folsäure [13].

Eine Ausnahme zu den negativen Studien bildet eine Untersuchung aus China (Linxian) an gesunden Männern 
und Frauen: Die Kombination aus Selen $(50 \mathrm{mg} / \mathrm{d}, 91 \%$ RDA), Vitamin E (30 mg/d, $250 \%$ RDA) und Beta-Carotin $(15 \mathrm{mg} / \mathrm{d}, 313 \%$ RDA) senkte die Gesamtmortalität um $10 \%$ [13]. Es muss jedoch kritisch angemerkt werden, dass diese Studie in einer ländlichen Region erfolgte, in der eine Unterversorgung mit Mikronährstoffen anzunehmen ist. Die hier gewonnenen Erkenntnisse können auf die mitteleuropäische Bevölkerung insofern nicht übertragen werden.

\section{Vitamin D}

Für Vitamin D bleibt die Datenlage trotz intensiver Forschung ebenfalls inkonsistent. Den größten positiven Effekt zeigte eine Studie bei 1179 gesunden Frauen über 55 Jahren, die eine tägliche Supplementation von Kalzium (1500 mg /d, $188 \%$ RDA) und Vitamin D (1100 IE/d, 550 \% RDA) erhielten. Das Risiko, an einem Tumor zu erkranken, war signifikant reduziert. Aufgrund der beeinträchtigten Studienqualität mit hoher Abbruchquote und weiterer, insbesondere negativer Studien (z. B. NHANES III [14]), empfehlen Bjelakovic et al. in einer Cochrane-Übersicht keine generelle Supplementation von Vitamin D zur Krebsvorbeugung [15].

\section{Multivitaminpräparate}

Da die Zusammensetzung und Dosierung von Multivitaminpräparaten nicht einheitlich definiert ist, können die nachfolgend dargestellten Studien nicht verglichen und ihre Ergebnisse nicht allgemein übertragen werden. Zwei große, beobachtende Studien wiesen keine Assoziation zwischen einer Einnahme von Multivitaminpräparaten und einer verminderten Krebsinzidenz nach [16, 17]. Eine große randomisierte, placebokontrollierte Studie an 14641 männlichen Ärzten (Physicians' Health Study II) zeigte hingegen eine kleine Risikoreduktion mit knapp signifikantem Ergebnis (Hazard Ratio [HR] 0,92; $95 \%$ Konfidenzintervall [95\%-KI] 0,860-0,998) [18]. Das untersuchte Multivitaminpräparat enthielt:

- Vitamin E (400 IU/d, $3000 \%$ RDA)

- Vitamin C (500 mg/d, $625 \%$ RDA)

- Beta-Carotin (50 mg/d, 1042 \% RDA)

Eine zweite große randomisierte, placebokontrollierte Studie zur selben Fragestellung untersuchte 13017 Probanden [19]. Das verwendete Multivitaminpräparat enthielt fünf Substanzen:

- Vitamin C (120 mg/d, $150 \%$ RDA)

- Vitamin E (30 mg/d, $250 \%$ RDA)

- Beta-Carotin (6 mg/d, $125 \%$ RDA)

- Selen $(100 \mu \mathrm{g} / \mathrm{d}, 182 \%$ RDA $)$

- Zink (20 mg/d, $200 \%$ RDA)
Es zeigte sich ein positiver Effekt bei den 5141 männlichen Probanden (relatives Risiko 0,69; $95 \%$ KI 0,53$0,91)$, jedoch nicht bei den 7876 Teilnehmerinnen. Der Interaktionstest war negativ $(p=0.11)$.

Aufgrund der eingangs erwähnten Limitationen, der inkonsistenten Datenlage sowie der teilweise geringen Effekte einer Supplementation, wird in einer kürzlich erschienenen Metaanalyse eine Primärprävention mit Multivitaminpräparaten derzeit nicht empfohlen [20].

\section{Merke}

In Anbetracht aller vorhandenen Studien kann generell keine Empfehlung für eine gezielte Einnahme von Mikronährstoffen zur Primärprävention von Tumorerkrankungen in Deutschland ausgesprochen werden $[6,7,21]$. Dies gilt auch für die Tertiärprävention nach kurativ behandelter Tumorerkrankung [21, 22]. Vielmehr ist als Prophylaxe einer Vielzahl von chronischen Erkrankungen eine vollwertige Ernährung reich an Gemüse und Obst empfehlenswert ( $\triangleright$ Abb. 1).

Eine vollwertige Ernährung kann beispielsweise auf den Empfehlungen der Deutschen Gesellschaft für Ernährung (DGE) beruhen [21]. Insbesondere in Kombination mit körperlicher Aktivität hat dies einen nachweislich protektiven Effekt [23 - 25], wobei die Effektstärke deutlich über einer rein „chemopräventiven“ Herangehensweise liegt. Dies gilt ebenfalls für das Vermeiden kanzerogener Noxen, wie dem Tabakrauch.

Es gilt anzumerken, dass für bestimmte Patientengruppen ein präventiver Effekt durch eine Supplementation von Mikronährstoffen prinzipiell möglich sein könnte [22, 26]. Weiterführende, prospektive Studien

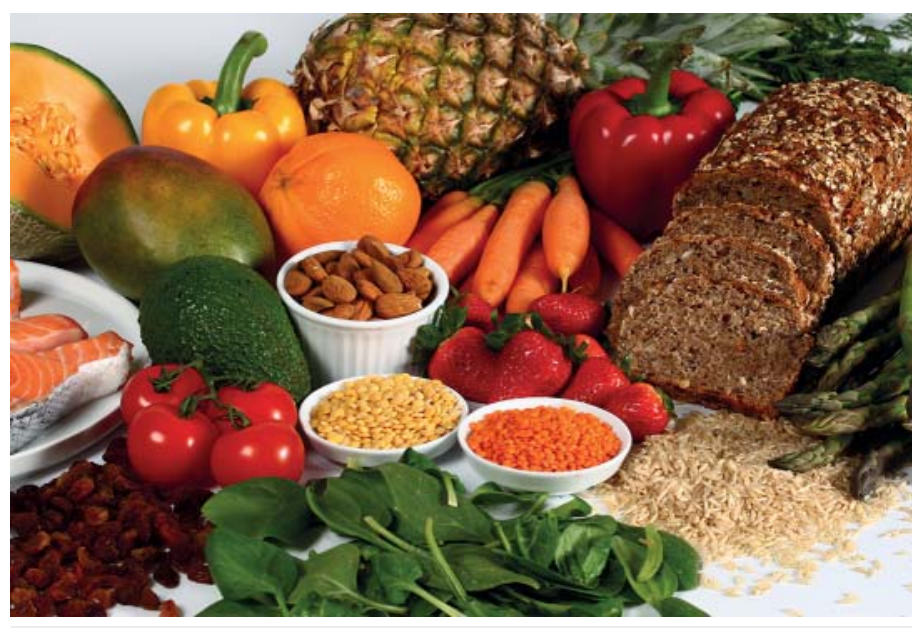

- Abb. 1 Eine vollwertige Ernährung reich an Obst und Gemüse in Kombination mit körperlicher Aktivität sollte zur Prävention von Krebserkrankungen empfohlen werden (Bildnachweis: Michael Zimmermann/Thieme Verlagsgruppe). 
müssen jedoch noch durchgeführt werden, um dies zu untersuchen.

\section{Vorbeugen und Beheben eines Mikronährstoffmangels bei onko- logischen Patienten}

\section{Mangelernährung bei onkologischen Patienten}

In Abhängigkeit von Tumorlokalisation, Histologie und Krankheitsstadium sind 30-90\% der onkologischen Patienten mangelernährt [27, 28]. Dies ist von großer klinischer Bedeutung, da nicht nur das Ansprechen auf antineoplastische Verfahren wie die Radio- und die Chemotherapie vermindert ist [29], sondern auch deren Nebenwirkungen verstärkt und die Lebensqualität sowie die Prognose des Patienten negativ beeinflusst werden können [30, 31].

\section{Mikronährstoffmangel bei onkologischen Patienten}

Eine Mangelernährung betrifft in der Regel nicht nur energieliefernde Nährstoffe, sondern auch Mikronährstoffe [21]. Ein gesteigerter Bedarf von Vitaminen und Spurenelementen im Sinne eines „Hypermetabolismus“ wird bei onkologischen Patienten zwar oft postuliert, derartige Befunde wurden beim Menschen bisher aber nicht objektiv erhoben [32]. Als Hinweis auf eine Mangelversorgung mit Mikronährstoffen werden oftmals Untersuchungen zitiert, die reduzierte Plasmaspiegel von Selen, Vitamin C und E sowie Vitamin D bei onkologischen Patienten aufzeigen [33, 34]. Hierbei muss jedoch kritisch angemerkt werden:

- Ein niedriger Blutspiegel zeigt nicht notwendigerweise und zuverlässig eine Mangelsituation für das jeweilige Vitamin an (das gilt insbesondere für Antioxidantien [32, 35]).

- Auch Symptome einer umschriebenen Hypovitaminose finden sich nicht so häufig wie reduzierte Plasmaspiegel einzelner Mikronährstoffe.

Entsprechend werden in der klinischen Praxis die Blutspiegel der meisten Antioxidantien nicht bestimmt und entsprechende Einzelstoffe nicht substituiert.

\section{Selen}

Eine Ausnahme unter den Antioxidantien stellt die Spiegelbestimmung von Selen dar. Allerdings ist ein Selenmangel ebenso wie allgemeingültige Referenzwerte für den Selen-Normbereich in der Literatur nicht einheitlich definiert. Als subklinischer Mangel wird meist ein Spiegel unter $75 \mu \mathrm{g}(0,95 \mu \mathrm{M})$ genannt [36].

Es besteht kein Konsens darüber, ob sich der SelenSerumspiegel als Marker für den Selenstatus eignet.
In zwei randomisierten Studien mit insgesamt 121 Teilnehmern wurde die Wirksamkeit von Natriumselenit zur Behandlung eines Selenmangels bei onkologischen Patienten untersucht $[37,38]$. Der Selenspiegel der Teilnehmer lag hierbei unter $85 \mu \mathrm{g} / \mathrm{l}$. Beide Studien untersuchten den Einfluss einer Selensubstitution auf radiogene Nebenwirkungen bei gynäkologischen Tumoren sowie Kopf-Hals-Karzinomen. Ein signifikanter Vorteil wurde lediglich bei Frauen mit Strahlentherapie der Beckenregion berichtet: Diarrhoen traten seltener auf [38].

Merke

Solange nicht mehr randomisierte klinische Studien sowie ein Konsensus zur einheitlichen Definition eines Selenmangels vorliegen, sollte nach individueller Risiko-Nutzen-Bewertung, zeitlich begrenzt und engmaschig kontrolliert eine entsprechende Substitution erwogen werden.

\section{Vitamin D}

Vitamin D wird - im Gegensatz zu den meisten Antioxidantien - in der klinischen Routine häufiger bestimmt, um einen Mangel zu erkennen. Bei einer Serumkonzentration $<20-30 \mathrm{ng} / \mathrm{ml}$ wird überwiegend von einem signifikanten Mangel ausgegangen [22, 39]. Allerdings hinterfragen einige Autoren diesen Grenzwert, insbesondere für gesunde Menschen [40]. Eine Spiegelbestimmung wird bei Verdacht auf Osteopenie oder Osteoporose empfohlen [39]. So lässt sich eine behandelbare Ursache erkennen und das Frakturrisiko abschätzen.

Merke

Welche onkologischen Patienten von einer VitaminD-Spiegelbestimmung mit ggf. hieraus resultierender Substitution profitieren, ist nicht hinreichend untersucht.

Auch wenn der Nutzen einer gezielten Vitamin-D-Substitution für onkologische Patienten noch nicht als bewiesen angesehen werden kann, so erscheint diese aufgrund der weiteren Gesundheitsvorteile, wie z. B. für die Knochengesundheit, erwägenswert [22]. Weiterhin könnte die Wirksamkeit von Bisphosphonaten, die oftmals bei Patienten mit Knochenmetastasen eingesetzt werden, bei einem Vitamin D-Spiegel über $33 \mathrm{ng} / \mathrm{ml}$ verbessert sein [41]. Auch die Wirksamkeit einer Therapie mit Rituximab könnte verbessert sein [42].

Merke

Sinnvoller als Einzelsubstanzen zu ersetzen ist es, eine Mangelernährung frühzeitig zu erkennen (z. B. mittels entsprechender Screeningverfahren, wie dem NRS-2002 [43]) und dieser mittels ausgewogener sowie vollwertiger Ernährung vorzubeugen. 
Als Richtlinie können auch hier die Empfehlungen der DGE betrachtet werden. Die S3-Leitlinie „Klinische Ernährung in der Onkologie“ der Deutschen Gesellschaft für Ernährungsmedizin (DGEM) empfiehlt ausdrücklich und mit starkem Konsens, dass die Ernährung von Tumorpatienten Vitamine und Spurenelemente in den gleichen Mengen enthalten soll, wie sie für gesunde Personen bzw. für künstliche Ernährung empfohlen werden [21]. Daher sollte bei Risikopatienten für eine Mangelernährung frühzeitig und regelmäßig eine qualifizierte onkologische Ernährungsberatung und ggf. eine ernährungsmedizinische Intervention erfolgen.

\section{Trink- und Zusatznahrung}

Bei eingeschränkter Nahrungsauswahl kann bei Tumorpatienten der Einsatz von Trink- und Zusatznahrung eine ausreichende Energie- und Mikronährstoffversorgung sicherstellen. Bei ausreichender Versorgung mit Makronährstoffen kann im Einzelfall der Einsatz eines Multivitamin-Multimineralsupplements mit physiologischen Dosierungen, d. h. Nährstoffmengen, die in etwa den
Tagesempfehlungen entsprechen, eine sinnvolle und sichere Maßnahme darstellen [31]. Dies gilt auch für onkologische Patienten während der Chemo- und Radiotherapie. In seltenen Fällen müssen Tumorpatienten auch parenteral ernährt werden ( $\triangleright$ Abb. 2).

\section{Beeinflussen von Effektivität und Nebenwirkungen onkologischer Therapien}

\section{Vitamin C}

Die meisten Untersuchungen liegen für Vitamin C vor. Präklinische Daten zeigen, dass eine hochdosierte Therapie mit Vitamin C antineoplastisch wirken könnte [44]. Entsprechend hohe Serumspiegel werden beim Menschen jedoch nur erreicht, wenn parenteral Dosierungen größer als $0,5 \mathrm{~g} / \mathrm{kg}$ Körpergewicht angewendet werden [45]. Mehrere Fallberichte und retrospektive Untersu-

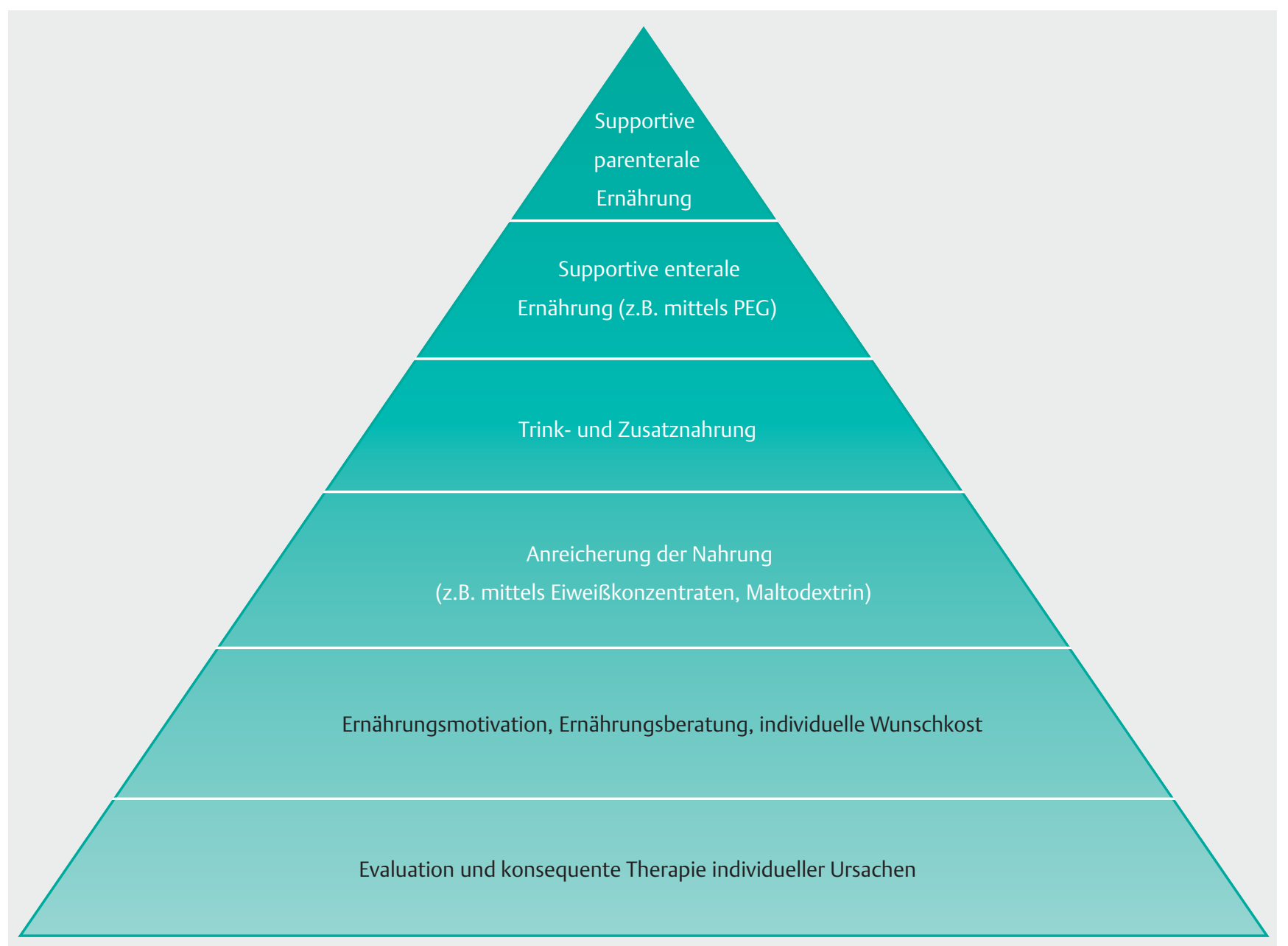

- Abb. 2 Stufenplan der Ernährungstherapie [52]. 
chungen zeigen einen positiven Effekt einer hochdosierten, parenteralen Gabe von Vitamin C auf

- Lebensqualität,

- Nebenwirkungen antineoplastischer Therapien sowie

- teilweise Tumoransprechen und Überleben [3, 31, $46,47]$.

Kontrollierte, prospektive Interventionsstudien sind derzeit jedoch nur wenige mit überwiegend kleinen Patientenzahlen vorhanden (meist Phase I/II-Studien). Die einzige kontrollierte und randomisierte Studie stammt von Ma et al. und untersuchte an 27 Patientinnen mit Ovarialkarzinom im Stadium III/IV parallel zur konventionellen Chemotherapie mit Carboplatin und Paclitaxel die intravenöse Gabe von Vitamin C [45]. Es zeigte sich eine reduzierte Toxizität der verwendeten Chemotherapie. Um die Wirksamkeit zu evaluieren und eine hochdosierte Vitamin C-Substitution zu empfehlen, sollten weiterführende, kontrollierte sowie randomisierte Studien abgewartet werden.

\section{Selen}

Zum Einsatz von Selen liegen nur kleine randomisierte, jedoch nicht verblindete und nicht placebokontrollierte Studien vor [3, 48]. Diese zeigen einen positiven Einfluss auf verschiedene Nebenwirkungen antineoplastischer Therapien, wie z. B. Fatigue, Hämatotoxizität oder Alopezie. Aufgrund der kleinen Fallzahl, der Studienqualität sowie potenzieller Nebenwirkungen einer Selenüberdosierung empfehlen die Autoren einer Übersichtsarbeit, Selen in der Behandlung von Tumorerkrankungen nicht generell zu befürworten [21, 49].

\section{Wechselwirkungen}

Die Wechselwirkung zwischen oxidativem Stress und den erwünschten als auch unerwünschten Wirkungen antineoplastischer Therapien ist überwiegend nicht verstanden [21]. Für einige Zytostatika wurde die Induktion von oxidativem Stresses als Wirkmechanismus beschrieben. In diesem Zusammenhang berichten mehrere Arbeiten, dass diese Zytostatika bei Kombination mit Antioxidantien vermindert wirken [21, 50, 51]. Der Einsatz von Antioxidantien ist seither umstritten [31].

\footnotetext{
Merke
In Anbetracht des noch unbestätigten Nutzens einer hochdosierten Behandlung mit Antioxidantien sowie potenzieller Wechselwirkungen mit antineoplastischen Therapien empfiehlt die DGEM in der aktuellen S3-Leitlinie mit starkem Konsens, Antioxidantien während einer Chemotherapie nicht hochdosiert zu geben [21].
}

\section{Etablierte Vitamingaben in der Onkologie}

Von einer hochdosierten Gabe von Antioxidantien abgesehen, ist die hochdosierte Gabe von weiteren Vitaminen in bestimmten Indikationen innerhalb der modernen Onkologie etabliert. So steigert aktivierte Folsäure (Folinsäure) die Aktivität des Zytostatikums Fluorouracil. Eine entsprechende Kombination wird heutzutage für eine Vielzahl, insbesondere gastrointestinaler Tumore, eingesetzt (z. B. in Kombination mit Irinotecan im FOLFIRISchema oder Oxaliplatin im FOLFOX-Schema).

Weiterhin wird Vitamin B12 und Folsäure begleitend zum Zytostatikum Pemetrexed, einem Folsäureantagonisten, gegeben (siehe Fachinformation). Hiermit werden Nebenwirkungen deutlich reduziert. Schließlich wird Folinsäure in vergleichbarer Indikation bei Hochdosistherapien mit dem Folsäureantagonisten Methotrexat eingesetzt.

\section{KERNAUSSAGEN}

- Die Einnahme von Mikronährstoffen zur Vorbeugung von Krebs kann nach aktueller Evidenzlage nicht empfohlen werden.

- Eine effiziente Risikoreduktion bezüglich Krebsentstehung kann erreicht werden durch eine gesunde und abwechslungsreiche Ernährung, regelmäßiger körperlicher Aktivität sowie durch Meiden von kanzerogenen Noxen.

- Einer möglichen Mangelernährung onkologischer Patienten sollte frühzeitig und umfassend entgegengewirkt werden anstatt Einzelsubstanzen zu ersetzen.

- Der Ausgleich eines meist subklinischen Mangels an Vitamin D sollte nach individueller Risiko-Nutzen-Bewertung abgewogen werden.

- Eine hochdosierte Gabe von Antioxidantien kann unter Chemotherapie nicht empfohlen werden.

\section{Interessenkonflikte}

JWH: Vortragshonorare von Roche MM: keine

VH: Honorare, Reisekosten und/oder Mitgliedschaft in Advisory Boards von Merck, Roche, Amgen, Sanofi, Sirtex, Baxaltza, Lilly, SIRTEX, Böhringer Ingelheim, Taiho, Merrimack. NE: Vortragshonorare von CSL Behring; B-Braun. Autorenhonorar von Klarigo. 


\section{맘 \\ Dr. med. Julian Walter Holch \\ ist Assistenzarzt, Ernährungsmediziner und wissenschaftlicher Mitarbeiter an der Medizini- schen Klinik und Poliklinik III, Klinikum der Uni- versität München, Campus Großhadern, sowie am Comprehensive Cancer Center München. julian.holch@med.uni-muenchen.de}

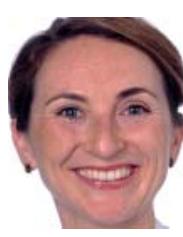

\section{Dr. med. Marlies Michl}

ist Fachärztin für Innere Medizin, Hämatologie und Onkologie und wissenschaftliche Mitarbeiterin an der Medizinischen Klinik und Poliklinik III, Klinikum der Universität München, Campus Großhadern sowie am Comprehensive Cancer Center München.

marlies.michl@med.uni-muenchen.de

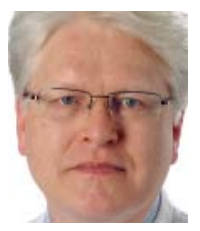

\section{Prof. Dr. med. Volker Heinemann}

ist Oberarzt an der Medizinischen Klinik und Poliklinik III, Klinikum der Universität München, Campus Großhadern sowie Direktor des Comprehensive Cancer Center der LMU - Krebszentrum München.

volker.heinemann@med.uni-muenchen.de

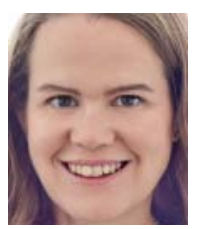

\section{Nicole Erickson, MS.c., RD}

ist als Ernährungswissenschaftlerin am Comprehensive Cancer Center München, Klinikum der Universität München tätig. Nicole.Erickson@med.uni-muenchen.de

\section{Korrespondenzadresse}

Dr. med. Julian Walter Holch

Medizinische Klinik und Poliklinik III, Klinikum der Universität München

Campus Großhadern

81377 München

julian.holch@med.uni-muenchen.de

\section{Literatur}

[1] Molassiotis A, Fernadez-Ortega P, Pud D et al. Use of complementary and alternative medicine in cancer patients: a European survey. Ann Oncol 2005; 16: 655-663

[2] Velicer CM, Ulrich CM. Vitamin and mineral supplement use among US adults after cancer diagnosis: a systematic review. J Clin Oncol 2008; 26: 665-673

[3] Gröber U, Mücke R, Adamietz IA. Komplementärer Einsatz von Antioxidanzien und Mikronährstoffen in der Onkologie. Onkologe 2013; 18: 136

[4] Valko M, Rhodes CJ, Moncol J. Free radicals, metals and antioxidants in oxidative stress-induced cancer. Chem Biol Interact 2006; 160: 1 - 40

[5] Willcox JK, Ash SL, Catignani GL. Antioxidants and prevention of chronic disease. Crit Rev Food Sci Nutr 2004; 44: 275-295

[6] Bjelakovic G, Nikolova D, Gluud LL et al. Antioxidant supplements for prevention of mortality in healthy participants and patients with various diseases. Cochrane Database Syst Rev 2012. DOI: CD007176

[7] Vinceti M, Dennert G, Crespi CM et al. Selenium for preventing cancer. Cochrane Database Syst Rev 2014. DOI: CD005195

[8] The Alpha-Tocopherol, Beta Carotene Cancer Prevention Study Group. The effect of vitamin $\mathrm{E}$ and beta carotene on the incidence of lung cancer and other cancers in male smokers. N Engl J Med 1994; 330: 1029-1035

[9] Goodman GE, Thornquist MD, Balmes J et al. The Beta-Carotene and Retinol Efficacy Trial: incidence of lung cancer and cardiovascular disease mortality during 6-year follow-up after stopping beta-carotene and retinol supplements. J Natl Cancer Inst 2004; 96: $1743-1750$

[10] Lippman SM, Klein EA, Goodman PJ et al. Effect of selenium and vitamin $\mathrm{E}$ on risk of prostate cancer and other cancers: the Selenium and Vitamin E Cancer Prevention Trial (SELECT). JAMA 2009; 301: 39-51

[11] Klein EA, Thompson IM, Tangen CM et al. Vitamin E and the risk of prostate cancer: the Selenium and Vitamin E Cancer Prevention Trial (SELECT). JAMA 2011; 306: 1549-1556

[12] Miller ER, Pastor-Barriuso R, Dalal D et al. Meta-analysis: high-dosage vitamin E supplementation may increase allcause mortality. Ann Intern Med 2005; 142: 37 - 46

[13] Ebbing M, Bonaa KH, Nygard O et al. Cancer incidence and mortality after treatment with folic acid and vitamin B12. JAMA 2009; 302: 2119-2126

[14] Freedman DM, Looker AC, Abnet CC et al. Serum 25-hydroxyvitamin D and cancer mortality in the NHANES III study (19882006). Cancer Res 2010; 70: 8587-8597

[15] Bjelakovic G, Gluud LL, Nikolova D et al. Vitamin D supplementation for prevention of cancer in adults. Cochrane Database Syst Rev 2014. DOI: CD007469

[16] Neuhouser ML, Wassertheil-Smoller S, Thomson C et al. Multivitamin use and risk of cancer and cardiovascular disease in the Women's Health Initiative cohorts. Arch Intern Med 2009; 169: $294-304$

[17] Park SY, Murphy SP, Wilkens LR et al. Multivitamin use and the risk of mortality and cancer incidence: the multiethnic cohort study. Am J Epidemiol 2011; 173: 906 - 914

[18] Gaziano JM, Glynn RJ, Christen WG et al. Vitamins E and C in the prevention of prostate and total cancer in men: the Physicians' Health Study II randomized controlled trial. JAMA 2009; 301: $52-62$

[19] Hercberg S, Galan P, Preziosi P et al. The SU.VI.MAX Study: a randomized, placebo-controlled trial of the health effects of antioxidant vitamins and minerals. Arch Intern Med 2004; 164: $2335-2342$

[20] Fortmann SP, Burda BU, Senger CA et al. Vitamin and mineral supplements in the primary prevention of cardiovascular disease and cancer: An updated systematic evidence review for the U.S. Preventive Services Task Force. Ann Intern Med 2013; 159: $824-834$

[21] Arends JBH, Bischoff SC, Fietkau R et al. S3-Leitline Klinische Ernährung in der Onkologie. Aktuel Ernahrungsmed 2015; 40: e1-e74

[22] Giovannucci E, Chan AT. Role of vitamin and mineral supplementation and aspirin use in cancer survivors. J Clin Oncol 2010; 28: $4081-4085$

[23] Anderson AS, Craigie AM, Caswell S et al. The impact of a bodyweight and physical activity intervention (BeWEL) initiated through a national colorectal cancer screening programme: randomised controlled trial. BMJ 2014; 348: g1823 
[24] Patel AV, Feigelson HS, Talbot JT et al. The role of body weight in the relationship between physical activity and endometrial cancer: results from a large cohort of US women. Int J Cancer 2008; 123: $1877-1882$

[25] Gibson TM, Park Y, Robien K et al. Body mass index and risk of second obesity-associated cancers after colorectal cancer: a pooled analysis of prospective cohort studies. J Clin Oncol 2014; 32: 4004-4011

[26] Biesalski HK, Grune T, Tinz ] et al. Reexamination of a metaanalysis of the effect of antioxidant supplementation on mortality and health in randomized trials. Nutrients 2010; 2: $929-$ 949

[27] Bozzetti F. Screening the nutritional status in oncology: a preliminary report on 1,000 outpatients. Support Care Cancer 2009; 17: 279-284

[28] Pirlich M, Schutz T, Norman K et al. The German hospital malnutrition study. Clin Nutr 2006; 25: 563-572

[29] Ross PJ, Ashley S, Norton A et al. Do patients with weight loss have a worse outcome when undergoing chemotherapy for lung cancers? Br J Cancer 2004; 90: 1905-1911

[30] Marin Caro MM, Laviano A, Pichard C. Impact of nutrition on quality of life during cancer. Curr Opin Clin Nutr Metab Care 2007; 10: $480-487$

[31] Strohle A, Zanker K, Hahn A. Nutrition in oncology: the case of micronutrients (review). Oncol Rep 2010; 24: 815-828

[32] Biesalski HK. Mikronaehrstoffsupplemente bei onkologischen Patienten. Aktuel Ernahrungsmed 2011; 36: 282 - 285

[33] Skrzydlewska E, Sulkowski S, Koda M et al. Lipid peroxidation and antioxidant status in colorectal cancer. World J Gastroenterol 2005; 11: $403-406$

[34] Crew KD, Shane E, Cremers S et al. High prevalence of vitamin $\mathrm{D}$ deficiency despite supplementation in premenopausal women with breast cancer undergoing adjuvant chemotherapy. J Clin Oncol 2009; 27: 2151 - 2156

[35] Anthony HM, Schorah C]. Severe hypovitaminosis C in lungcancer patients: the utilization of vitamin $\mathrm{C}$ in surgical repair and lymphocyte-related host resistance. $\mathrm{Br}$ J Cancer 1982; 46: $354-367$

[36] Arnaud J, Bertrais S, Roussel AM et al. Serum selenium determinants in French adults: the SU.VI.M.AX study. Br J Nutr 2006; 95: $313-320$

[37] Buntzel ], Riesenbeck D, Glatzel M et al. Limited effects of selenium substitution in the prevention of radiation-associated toxicities. results of a randomized study in head and neck cancer patients. Anticancer Res 2010; 30: 1829-1832

[38] Muecke R, Schomburg L, Glatzel M et al. Multicenter, phase 3 trial comparing selenium supplementation with observation in gynecologic radiation oncology. Int J Radiat Oncol Biol Phys 2010; 78: $828-835$
[39] Bischoff-Ferrari HA, Giovannucci E, Willett WC et al. Estimation of optimal serum concentrations of 25-hydroxyvitamin D for multiple health outcomes. Am J Clin Nutr 2006; 84: 18 - 28

[40] Manson JE, Brannon PM, Rosen CJ et al. Vitamin D Deficiency Is There Really a Pandemic? N Engl J Med 2016; 375: 1817 1820

[41] Carmel AS, Shieh A, Bang $\mathrm{H}$ et al. The 25(OH)D level needed to maintain a favorable bisphosphonate response is $>1=33 \mathrm{ng} / \mathrm{ml}$. Osteoporos Int 2012; 23: 2479-2487

[42] Bittenbring JT, Neumann F, Altmann B et al. Vitamin D deficiency impairs rituximab-mediated cellular cytotoxicity and outcome of patients with diffuse large B-cell lymphoma treated with but not without rituximab. J Clin Oncol 2014; 32: $3242-3248$

[43] Kondrup J, Rasmussen $\mathrm{HH}$, Hamberg $\mathrm{O}$ et al. Nutritional risk screening (NRS 2002): a new method based on an analysis of controlled clinical trials. Clin Nutr 2003; 22: 321 - 336

[44] van der Reest J, Gottlieb E. Anti-cancer effects of vitamin C revisited. Cell Res 2016; 26: $269-270$

[45] Ma Y, Chapman J, Levine $\mathrm{M}$ et al. High-dose parenteral ascorbate enhanced chemosensitivity of ovarian cancer and reduced toxicity of chemotherapy. Sci Transl Med 2014; 6: DOI: 222ra18

[46] Vollbracht C, Schneider B, Leendert V et al. Intravenous vitamin $C$ administration improves quality of life in breast cancer patients during chemo-/radiotherapy and aftercare: results of a retrospective, multicentre, epidemiological cohort study in Germany. In Vivo 2011; 25: 983 - 990

[47] Drisko JA, Chapman J, Hunter VJ. The use of antioxidants with first-line chemotherapy in two cases of ovarian cancer. J Am Coll Nutr 2003; 22: $118-123$

[48] Vieira ML, Fonseca FL, Costa LG et al. Supplementation with selenium can influence nausea, fatigue, physical, renal, and liver function of children and adolescents with cancer. J Med Food 2015; 18: 109-117

[49] Dennert G, Horneber M. Selenium for alleviating the side effects of chemotherapy, radiotherapy and surgery in cancer patients. Cochrane Database Syst Rev 2006. DOI: CD005037

[50] Heaney ML, Gardner JR, Karasavvas N et al. Vitamin C antagonizes the cytotoxic effects of antineoplastic drugs. Cancer Res 2008; 68: $8031-8038$

[51] Perrone G, Hideshima T, Ikeda $\mathrm{H}$ et al. Ascorbic acid inhibits antitumor activity of bortezomib in vivo. Leukemia 2009; 23: $1679-1686$

[52] Löser C. Malnutrition in hospital: the clinical and economic implications. Dtsch Ärztebl Int 2010; 107: 911 - 917

\section{Bibliografie}

Dol http://dx.doi.org/10.1055/s-0042-112046

Dtsch Med Wochenschr 2017; 142: 896-902

(c) Georg Thieme Verlag KG Stuttgart · New York ISSN 0012-0472 